\section{Commentary: Suture annuloplasty for isolated aortic valve repair: The Holy Grail is yet to be found}

\author{
François Dagenais, MD
}

In their article in this issue of the Journal, Federspiel and colleagues ${ }^{1}$ review current techniques for suture annuloplasty during isolated aortic valve repair. Since the initial descriptions of the reimplantation/remodeling techniques by David and Yacoub, significant milestones have been achieved in the field of aortic valve repair for aortic regurgitation. Although initially viewed as more "art" than "science," significant contributions have led to a better understanding of the concepts of aortic valve repair. Foremost, as elegantly illustrated in the present report, thorough knowledge of the normal anatomy and physiology linked to aortic valve competency is essential. Efforts at standardizing procedures according to different root anatomies have led to a more comprehensive approach to the procedures, and hence better reproducibility. ${ }^{2}$ Finally, early echocardiographic findings, such as residual aortic regurgitation and effective leaflet height, have been identified as strong predictors of procedural outcome.

Annular dilatation often accompanies aortic regurgitation, especially in the presence of root dilatation. Although the need for concomitant annuloplasty during root remodeling procedures is a matter of debate, the general consensus is that annuloplasty is required in cases of an annulus size $>25$ to $27 \mathrm{~mm}$. A reimplantation technique has been shown to be effective for annular reduction and stabilization in the presence of root and annular dilatation. The ideal

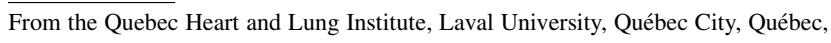
Canada.

Disclosures: Dr Dagenais serves as a consultant and proctor for COOK Medical, Edwards Lifesciences, and Medtronic.

The Journal policy requires editors and reviewers to disclose conflicts of interest and to decline handling or reviewing manuscripts for which they may have a conflict of interest. The editors and reviewers of this article have no conflicts of interest.

Received for publication Jan 23, 2021; revisions received Jan 23, 2021; accepted for publication Jan 25, 2021; available ahead of print Jan 30, 2021.

Address for reprints: François Dagenais, MD, Institut Universitaire de Cardiologie et de Pneumologie de Québec, 2725 Chemin Sainte-Foy, Québec, Québec, Canada G1V 4G5 (E-mail: francois.dagenais@chg.ulaval.ca).

JTCVS Techniques 2021;7:107-8

2666-2507

Copyright (C) 2021 The Authors. Published by Elsevier Inc. on behalf of The American Association for Thoracic Surgery. This is an open access article under the CC BY-NCND license (http://creativecommons.org/licenses/by-nc-nd/4.0/).

https://doi.org/10.1016/j.xjtc.2021.01.037

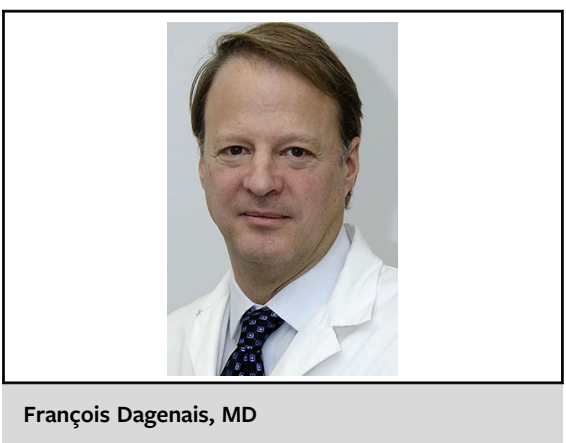

\section{CENTRAL MESSAGE \\ Although significant milestones \\ have been achieved in aortic \\ valve reconstruction, the ideal \\ annuloplasty technique in iso- \\ lated aortic valve repair remains \\ to be defined.}

annuloplasty technique in "isolated aortic valve repair" (aortic root $<40-45 \mathrm{~mm}$ ) not requiring root replacement is unclear and is the focus of the present review.

In an isolated aortic valve repair setting, the ideal annuloplasty technique should be effective in downsizing and stabilizing the annulus, easy to perform and reproducible, adapt to variability in annulus configurations, and able to restore/preserve root geometry. Furthermore, long-term durability with minimal complications, such as cusp mobility interference, conduction system anomalies, dehiscence, and biocompatibility, should be confirmed. Subcommissural sutures do not encompass the entire annulus and should not be considered a true annuloplasty. External rings have shown encouraging results with concomitant remodeling procedures. On the other hand, a variety of techniques using external, internal, or a combination of external and internal rings have been suggested for isolated aortic valve repair. Although the reported outcomes are acceptable, series remain small and limited to selected patients and few centers. Concerns remain about when to perform these procedures, as well as their reproducibility and adaptability to various root anatomies. The authors report the use of a polytetrafluoroethylene annuloplasty, a material shown to be durable in a mitral valve repair setting. The technique seems promising, because it can be tailored to the patient's anatomy, although a learning curve is reported to avoid impingement on surrounding structures. Thus, owing mainly to a lack of standardization and comparison among 
techniques, the authors conclude that with the current state of knowledge, no one technique can be recommended over another.

Great progress in the field of aortic valve repair has been made over the past 2 decades. In the presence of root dilatation, root remodeling/reimplantation procedures have evolved to be durable and reproducible techniques. The much less common isolated aortic valve repair procedures lack standardization. Further studies are needed to elucidate when and how to add an annuloplasty in conjunction with cusp repair in a nondilated root setting. The authors are to be congratulated for their ongoing leadership and innovation in this complex field of aortic surgery.

\section{References}

1. Federspiel J, Ehrlich T, Abeln K, Schäfers HJ. Aortic annuloplasty: subcommissural, intra-annular suture techniques, external and internal rings. J Thorac Cardiovasc Surg Tech. 2021;7:98-102.

2. Boodhwani M, de Kerchove L, Glineur D, Poncelet A, Rubay J, Astarci P, et al. Repair-oriented classification of aortic insufficiency: impact on surgical techniques and clinical outcomes. J Thorac Cardiovasc Surg. 2009;137:286-94. 